\title{
Innovative Interstellar Explorer: Radioisotope Propulsion to the Interstellar Medium
}

\author{
Ralph L. McNutt, Jr. , James C. Leary ${ }^{\dagger}$, Robert E. Gold ${ }^{\ddagger}$, Stamatios M. Krimigis ${ }^{\S}$, Edmond C. Roelof, \\ Johns Hopkins University Applied Physics Laboratory, Laurel, MD 20723-6099 \\ Mike Gruntman ${ }^{\dagger \dagger}$ \\ University of Southern California, Los Angeles, CA 90089-0182 \\ George Gloeckler ${ }^{\star}$, Patrick L. Koehn ${ }^{\S \S}$ \\ University of Michigan, Ann Arbor, MI 48109-2143 \\ William S. Kurth ${ }^{* * *}$ \\ The University of Iowa, Iowa City, IA 52242-1479 \\ Steven R. Oleson ${ }^{\dagger \dagger \dagger}$ \\ NASA Glenn Research Center, Cleveland, OH, 44135 \\ and \\ Douglas I. Fiehler ${ }^{\text {t* }}$ \\ QSS Group, Inc., NASA Glenn Research Center, Cleveland, OH 44135
}

\begin{abstract}
An interstellar "precursor" mission has been under discussion in the scientific community for over 25 years. Fundamental scientific questions about the interaction of the Sun with the interstellar medium can only be answered with in situ measurements that such a mission could provide. The Innovative Interstellar Explorer is a funded NASA Vision Mission Study that investigates the use of Radioisotope Electric Propulsion (REP) to enable such a mission. The problem is the development of a probe that can provide the required measurements and can reach a heliocentric distance of at least 200 astronomical units (AU) in a reasonable mission time. The required flyout speed in the direction of the inflowing interstellar medium is provided by a high-energy launch, followed by long-term, low-thrust, continuous acceleration. Trades from also using gravity assists have been studied along with trades between advanced Multi-mission radioisotope thermoelectric generators (MMRTGs) and Stirling radioisotope generators (SRGs), both powered by $\mathrm{Pu}$-238. While subject to mass and power limitations for the instruments on board, such an approach relies on known General Purpose Heat Source (GPHS), Pu-238 technology and current launch vehicles for
\end{abstract}

\footnotetext{
* Principal Professional Staff, Space Department, 11100 Johns Hopkins Road, MP3-E116, Member of AIAA

${ }^{\dagger}$ Section Supervisor, Space Department, 11100 Johns Hopkins Road, 4-154, Senior Member of AIAA

* Chief Technologist and Assistant Branch Supervisor, Space Department, 11100 Johns Hopkins Road, 23-306

$\S$ Emeritus Department Head, Space Department, 11100 Johns Hopkins Road, MP3-E134, Associate Fellow of AIAA

** Principal Professional Staff, Space Department, 11100 Johns Hopkins Road, MP3-E135

${ }^{\dagger}$ Professor of Astronautics, USC Viterbi School of Engineering, 854 Downey Way, RRB-224, MC-1192, Associate Fellow of AIAA

\$ Research Professor (and Professor of Physics, University of Maryland), Department of Atmospheric, Oceanic, and Space Sciences, 2455 Hayward St

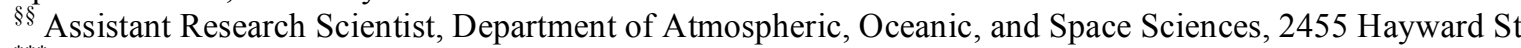

${ }^{* * *}$ Research Scientist, Department of Physics and Astronomy, 203 Van Allen Hall

${ }^{\dagger}$ Prometheus Electric Propulsion Segment Lead, 21000 Brookpark Rd., M.S. 301-3, Senior Member of AIAA

Electric Propulsion Systems Engineer, 21000 Brookpark Rd., M.S. 301-3, Member of AIAA
} 
speed, both of which require little new development and have well-known regulatory requirements for launch. The challenge is to assemble a fully self-consistent concept that addresses the science, instrument accommodation, propulsion, communications, guidance, and autonomy requirements for such a deep-space, long-lived mission. This approach avoids the intrinsically large masses associated with nuclear fission reactors and incorporates launch of all nuclear material directly into an Earth-escape trajectory. We discuss the science, payload, and development of this approach to an Interstellar Probe.

$\begin{array}{ll} & \text { Nomenclature } \\ \text { AU } & =1.495979 \times 10^{8} \mathrm{~km} \text {, Astronomical Unit, the mean distance from the Sun to the Earth } \\ \text { RPS } & =\text { Radioactive Power Source } \\ \text { RTG } & \text { Radioisotope Thermoelectric Generator } \\ \text { MMRTG } & =\text { Multi-mission Radioisotope Thermoelectric Generator } \\ \text { NEP } & =\text { Nuclear Electric Propulsion } \\ \text { NTP } & =\text { Nuclear Thermal Propulsion } \\ \text { REP } & =\text { Radioisotope Electric Propulsion } \\ \text { SRG } & =\text { Stirling Radioisotope Generator } \\ \text { Pu-238 } & =\text { plutonium isotope 238 } \\ \text { VLISM } & =\text { Very Local Interstellar Medium (region within } ~ 2000 \text { AU of the Sun) } \\ \text { MHD } & =\text { magnetohydrodynamic } \\ \mathrm{I}_{\mathrm{sp}} & =\text { specific impulse } \\ \mathrm{C}_{3} & =\text { excess (of escape) orbital energy per mass }\end{array}$

\section{Introduction}

$\mathrm{O}$ UR Sun emits a continuous supersonic, super-Alvenic stream of particles known as the solar wind. This flowing plasma, consisting primarily of protons, interacts with the Very Local Interstellar Medium (VLISM), producing a cavity in local interstellar space known as the heliosphere. The scale size of this region is dependent upon the properties of the VLISM, and details of the interaction ${ }^{1-5}$. We have known for some time that an "interstellar wind," also thought to be supersonic produces an asymmetry in the cavity, with the closest location interstellar medium in the direction of $+7^{\circ}$ declination and $252^{\circ}$ right ascension in Earth ecliptic coordinates ${ }^{2}$. To reach the interstellar medium in the shortest amount of time, a space probe needs to travel roughly in this direction. Our lack of knowledge of the properties of interstellar space, in particular of the magnetic field there has resulted in a great deal of speculation on the scale size of the interaction. Recently, the Voyager 1 spacecraft has returned data pointing to a crossing of the "termination shock" of the solar wind, with the cleanest signature on day 350 of 2004 at an Earth ecliptic latitude of $34.1^{\circ}$ and a heliocentric, i.e., Sun-centered, distance of 94.01 AU. Beyond the shock is the "heliopause," the pressure-balance interface between the plasma components of the solar wind and of the interstellar medium. Further beyond that interface there may be an external shock before one reaches the "undisturbed" interstellar medium. The recent measurements by instrumentation on Voyager 1 suggest that the termination shock has unexpected properties that are still under study ${ }^{6}$. These properties suggest that magnetohydrodynamic (MHD) simulations of the magnetosphere that give a heliopause distance of $\sim 120$ AU may overestimate the true distance, if indeed a classic heliopause as such really exists.

The properties of the interstellar medium remain elusive. Remote sensing measurements rely upon distortions to stellar spectra to provide average properties of the medium, but only over distance of several light years. To actually measure the properties of the VLISM, especially the magnetic field and low-energy cosmic rays, both of which are excluded from the heliosphere, in situ sampling is required. A combination of this sampling with remote observations, can, for example, reveal the "clumpiness" of the interstellar medium.

There have been many formulations of a science rationale for an "interstellar precursor" mission that would not travel to a star, but would travel to the interstellar medium. Such a probe would explore the nature of that medium, the structure of the heliosphere (and its variations), and fundamental astrophysical properties that cannot be measured otherwise.

In this NASA-supported "Vision Mission" study, we consider the implementation of a scientifically compelling mission to the interstellar medium ${ }^{7}$. Dubbed the "Innovative Interstellar Explorer," (IIE) the mission concept combines a compact payload, $\sim 1000-\mathrm{kg}$ (wet) class satellite, high launch energy, and electric propulsion. Four options are studied that combine various levels of risk and technical readiness for implementing this mission. 


\section{Requirements}

As with any robotic science mission, the true requirements flow from the science questions, though measurement objectives, required instruments, their required resources, mission and spacecraft requirements, data products, analysis products, and finally closure of the science questions. For this mission, all of these aspects are subject to the drivers of reaching a certain distance within a set time and providing for the return of the necessary data. In the original Vision Mission call for proposals, the NASA goal is to reach 200 AU (set by the scale size of the heliosphere) within 15 years (set by a "professional lifetime"). In addition, we set a minimum data return requirement of 500 bits per second (bps) based upon similar measurement requirements from spacecraft operating near Earth and other planets of the solar system. A derived "requirement" is that the spacecraft must function in the environment out to $200 \mathrm{AU}$ from the Sun.

\section{A. Science Requirements}

NASA's Interstellar Probe Science and Technology Definition Team (IPSTDT) discussed science requirements for an interstellar precursor mission were in 1999. In addition, the 2003 NASA Strategic Plan (operative at the beginning of this work) included: "NASA Strategic Plan Objective 5.13: Understand the changing flow of energy and matter throughout the Sun, heliosphere, and planetary environments. The dynamics of the solar system define the environment in which we live. These dynamics include the origins of variability in the Sun and solar wind, how the magnetospheres and atmospheres of Earth and other planets respond now and have evolved, and how the outermost region of space influenced by our star interacts with the rest of the galaxy of which it is a part...." [Emphasis added]. Together with IPSTDT, this frames the Science Traceability Matrix for such missions (Table 1).

In many deliberations, a payload consisting of nine instruments was considered both sufficient and necessary for carrying out a comprehensive mission: magnetometer (MAG), plasma wave sensor (PWS), plasma spectrometer (PLS), Energetic particle spectrometer (EPS), cosmic-ray spectrometer: anomalous cosmic rays (CRS-ACR), galactic cosmic rays (CRS-GCR), and electrons/positrons, protons, helium (CRS-LoZCR), cosmic dust sensor (CDS), neutral atom detector (NAD), energetic neutral atom imager (ENA), and a Lyman-alpha detector (LAD). Such a payload does leave out the possibility of measuring global dust distribution from the spacecraft platform (light-grey font in Table 1). Implementation of such a capability requires an infrared imaging telescope in addition to more requirements on pointing than imposed by the remainder of the payload. The infrared telescope itself was found to be a mass driver for a low-mass payload. Hence, at the top-level of the systems trade space, this instrument - and remote dust measurements - were deleted from further consideration.

\section{B. Payload Requirements}

In order to implement the mission, a light weight, low-power payload that can still accomplish the required measurements is required. We can make some estimates of the available mass and required power from considering similar instruments on similarly sized spacecraft. As a rough estimate, the spacecraft wet mass should be $\sim 1000 \mathrm{~kg}$. If half of the wet mass is propellant, then the dry mass is $\sim 500 \mathrm{~kg}$. Historically, instrument payloads are $\sim 5$ to $10 \%$ of the dry mass; hence, we can estimate that the allowable payload is in the range $\sim 25$ to $50 \mathrm{~kg}$. Spacecraft instrumentation typically uses $\sim 1 \mathrm{~W} / \mathrm{kg}$, so we also estimate a payload usage of $\sim 25$ to $50 \mathrm{~W}$. The study by the IPSTDT resulted in a notional payload of thirteen instruments with a mass of $26.6 \mathrm{~kg}$ and requiring $19.3 \mathrm{~W}$ of power. The nominal data rate for that payload is $24.6 \mathrm{bps}$. Comparison of ideal instruments currently flying on spacecraft where mass was not such a driver gives a total mass and power requirement of about $220 \mathrm{~kg}$ and $240 \mathrm{~W}$. Again, in looking at the overall system, we have assembled a notional payload for IIE that can make the required measurements to answer the science questions of Table 1. This instrument table is shown in Table 2. The following points should be noted: (1) instrument acronyms link the entries in Tables 1 and 2, (2) the implicit assumption is made that the IIE spacecraft is a spinner, with a spin rate of at least once per day. This enables the various instruments of the payload to scan the entire sky, as needed, without additional scan platforms, and (3) sufficient onboard processing is assumed to allow the down-linked data rate and duty cycle of the spacecraft to be matched to the (much higher) rate of data capture that is possible with the payload. 
Table 1. Science Traceability Matrix for the Innovative Interstellar Explorer

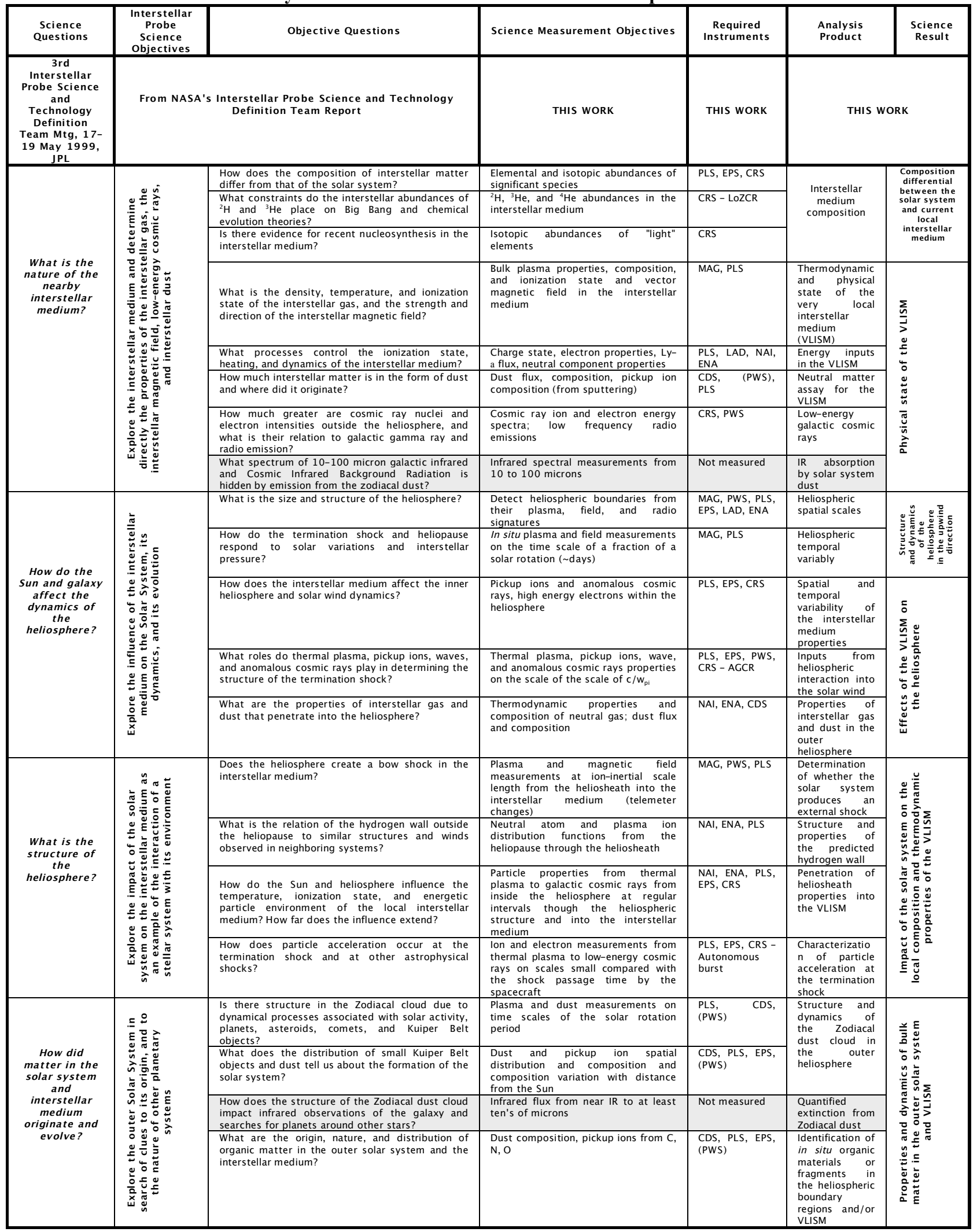

American Institute of Aeronautics and Astronautics 
Table 2. Instrument Resources, Capabilities, and Requirements

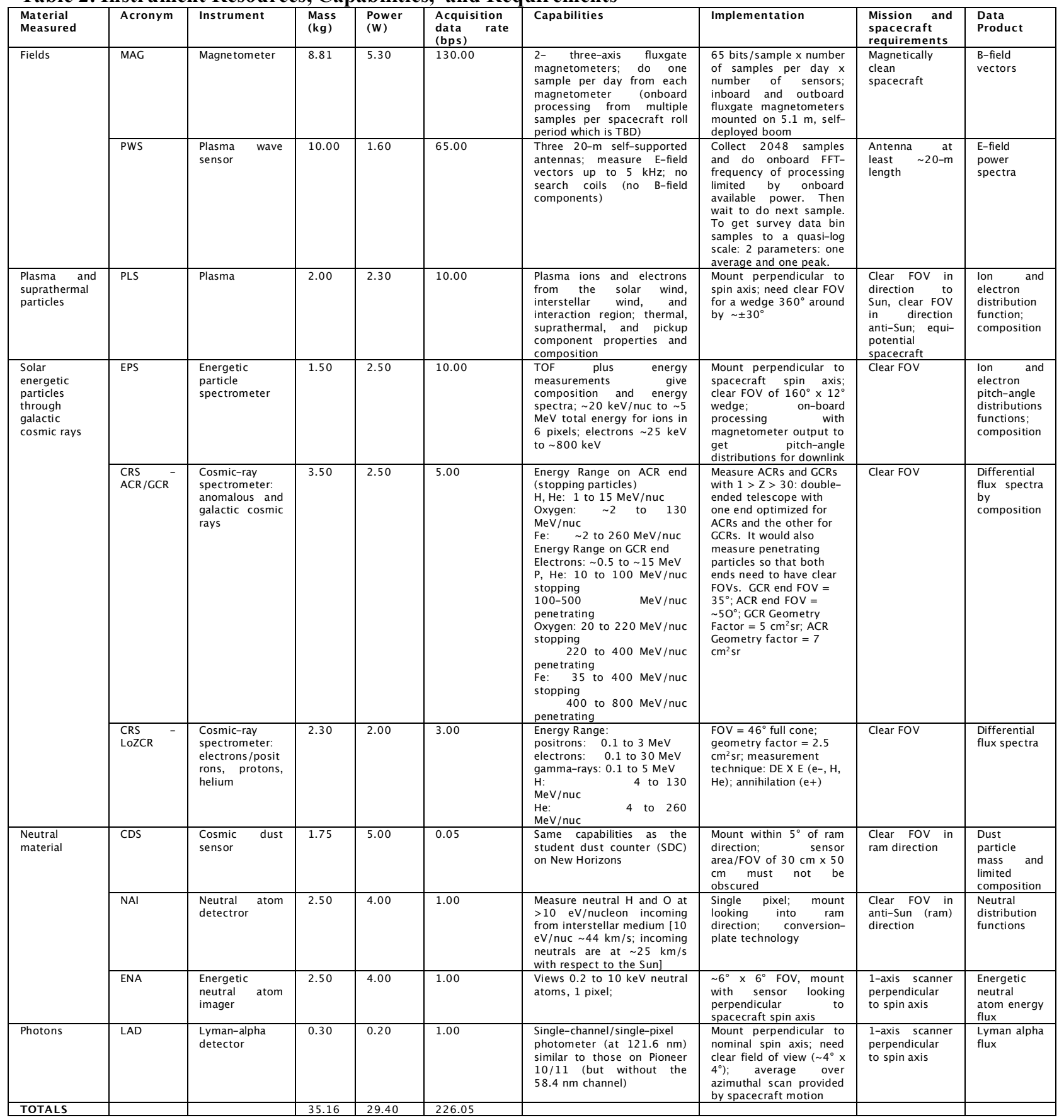

\section{Engineering Requirements}

The engineering requirements for all interstellar precursor missions studied can be summed up as "reach the interstellar medium as rapidly as possible." Reaching $200 \mathrm{AU}$ in 15 years is an average speed of $\sim 13 \mathrm{AU} / \mathrm{yr}$ or 63 
$\mathrm{km} / \mathrm{s}$, just more than twice Earth's orbital speed. Such large velocities cannot be practically achieved with any chemical rocket or even nuclear thermal propulsion (NTP). Previous studies have focused on ballistic missions using powered near-Sun gravity assists ${ }^{8-14}$, nuclear electric propulsion (NEP) ${ }^{15-20}$, and solar sail propulsion ${ }^{21-23}$. The first approach (ballistic) has significant thermal issues and still requires a non-conventional propulsion system near the Sun to reach the desired speeds. As an example to reach a solar system escape speed of 15 AU/yr with a maneuver applied at 4 solar radii (measured from the Sun's center), the implied $\Delta \mathrm{V}$ is $\sim 8.2 \mathrm{~km} / \mathrm{s}$. The second approach (NEP) provides inherently large systems that require power system mass-to-power systems of less than $\sim 30 \mathrm{~kg} / \mathrm{kW}$ to have the required capability. The third approach (solar sails) requires large ( $\sim 400-\mathrm{m}$ dia) highperformance $\left(\sim 1 \mathrm{~g} / \mathrm{m}^{2}\right)$ sails and small $(\sim 250 \mathrm{~kg})$ spacecraft and science payloads. This is the first detailed study of radioisotope electric propulsion (REP) for such a mission ${ }^{24}$.

For the top-level requirements for the mission we adopt the following:

Asymptotic trajectory within a $20^{\circ}$ cone of the "heliospheric nose" $\left(+7^{\circ}, 252^{\circ}\right.$ Earth ecliptic coordinates $)$

Provide data from $10 \mathrm{AU}$ to $200 \mathrm{AU}$

Arrive at $200 \mathrm{AU}$ "as fast as possible"

Consider all possible missions that launch between 2010 and 2050

Use existing launch hardware

No "in-space" assembly

Launch to escape velocity

Keep new hardware and technology to a minimum

Provide accepted "adequate" margins

These requirements leave open the transit time to $200 \mathrm{AU}$ as an optimization variable while minimizing new required technology and infrastructure.

\section{Mission Design}

To bracket fully the set of all possible mission scenarios we used an initial dry mass estimate of $519 \mathrm{~kg}$ and used this to explore the mission space for a variety of scenarios between 2010 and 2050. In each case, the optimized parameters included the launch $\mathrm{C}_{3}$, mass of xenon propellant for the REP electric propulsion (EP) system, the specific impulse of that engine, and its run time. Input engine power was fixed. A variety of planetary flyby missions were considered as well as a "direct-ascent" trajectory. Only outer planets were considered for planetary gravity assists, and all of these were un-powered. Earth gravity assists were not considered due to the presence of $\mathrm{Pu}-238$ in the power supplies and Venus flybys were not considered due to the additional thermal considerations. In all cases, ion engine use to line up the incoming flyby asymptote of the trajectory was part of the optimization. Hence, we did not look at (chemically-powered) gravity assists due to the extra complexity that is associated with the required configuration.

\section{A. Direct Trajectory}

A launch opportunity was identified on February 3, 2010, with a 3month launch window extending from December 7, 2009 to March 22, 2010. In this scenario, the EP system would operate at $52 \%$ efficiency on an input power of $1000 \mathrm{~W}$ and an $I_{\mathrm{sp}}$ of $2559 \mathrm{sec}$. This low-thrust system would burn for $\sim 26$ years, boosting the delta $\mathrm{V}$ by $32.4 \mathrm{~km} / \mathrm{s}$, until the probe reached $66 \mathrm{AU}$ (on April 19, 2036). Thereafter, the probe would then coast to $200 \mathrm{AU}$, reaching its destination 46.5 years after launch (Figure 1). The launch vehicle (including upper stage boosters) would need to provide a $\mathrm{C}_{3}$ of $103.8 \mathrm{~km}^{2} / \mathrm{s}^{2}$ to lift an $1885-\mathrm{kg}$ probe (wet mass) to a solar system escape trajectory. This probe would need to consume $1366 \mathrm{~kg}$ of xenon propellant during EP operation in order to deliver a $519-\mathrm{kg}$ spacecraft to the interstellar medium. This trajectory was not considered viable because of the long flight time and large xenon propellant mass (and thus complex EP system) that would be needed.

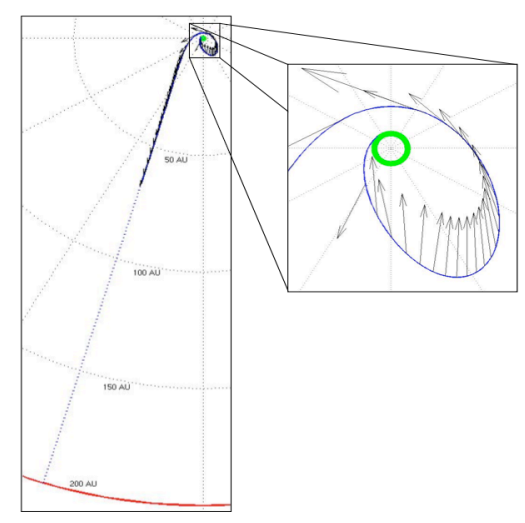

Figure 1. Direct-ascent trajectory. 


\section{B. Single-planet Gravity Assist}

To increase the asymptotic escape speed from the solar system, several outer planet gravity assists (Jupiter, Saturn, Uranus, and Neptune) were considered. The Jupiter gravity assist was chosen as the baseline because of the low launch mass and $<30$ year mission duration.

A Saturn gravity assist (SGA) trajectory would reduce the needed xenon propellant mass down to $932 \mathrm{~kg}$ (a factor of $\sim 1.5$ reduction). The $1451 \mathrm{~kg}$ probe would need to be launched on August 26, 203 in order to reach Saturn $\sim 3$ years later. The EP system (at $2800 \mathrm{sec} \mathrm{I}_{\mathrm{sp}}$ and $52.5 \%$ efficiency) would operate for 21.2 years to add a delta $\mathrm{V}$ of $28.2 \mathrm{~km} / \mathrm{s} .200 \mathrm{AU}$ would be reached after 33.5 years. The late launch date rendered this option undesirable.

Uranus gravity assist (UGA): Launching on August 25, 2035 to a total $\mathrm{C}_{3}$ of $122 \mathrm{~km}^{2} / \mathrm{s}^{2}$, the probe would take $\sim 8$ years to reach Uranus $(12.8 \mathrm{~km} / \mathrm{s}$ gravity assist delta V). The EP system would thrust for 30 years up to $110 \mathrm{AU}$, and $200 \mathrm{AU}$ would be reached 42.1 years after launch. The marginal reduction in xenon propellant mass $(15 \mathrm{~kg}$ compared to SGA) and late launch/arrival dates rendered this option undesirable.

Neptune gravity assist (NGA): Launching on May 20, 2035 to a total $\mathrm{C}_{3}$ of $124.2 \mathrm{~km} 2 / \mathrm{s} 2$, the probe would take $\sim 20$ years to reach Neptune $(5.4 \mathrm{~km} / \mathrm{s}$ gravity assist delta V). The EP system would thrust for 43.7 year up to $122 \mathrm{AU}$, and $200 \mathrm{AU}$ would be reached 69 years after launch. This option only saved $59 \mathrm{~kg}$ of propellant while adding significantly to the flight time to $200 \mathrm{AU}$.

Jupiter gravity assist (JGA): This trajectory would reduce the needed xenon propellant mass down to $394 \mathrm{~kg}$ (more than a factor of 3), resulting in a wet spacecraft mass of only $913 \mathrm{~kg}$. The minimum trip time launch (to a $\mathrm{C}_{3}$ of $152.6 \mathrm{~km}^{2} / \mathrm{s}^{2}$ ) would occur on October 26,2014 , with a 2-week launch window (October 15, 2014 to November 3, 2014). The JGA would occur $\sim 13$ months after launch. The EP burnout distance would be 103 AU after 15 years of thrusting, and $200 \mathrm{AU}$ would be reached after $\sim 25$ years. This candidate trajectory met both the low launch mass and short flyout time requirements, despite exposure to the hostile radiation environment near Jupiter,. This mission design was selected for the baseline and used for additional analysis during the Team X study (Figure 2).

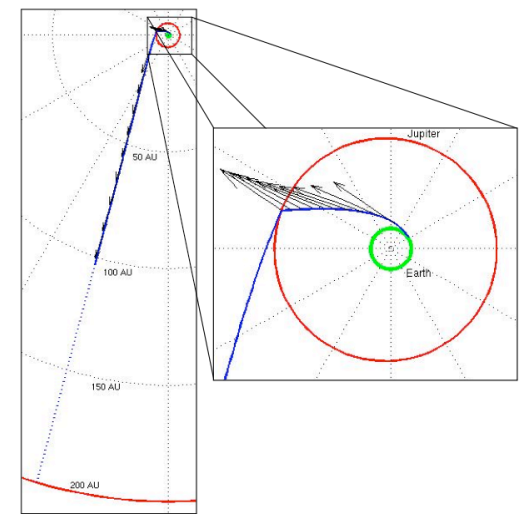

Figure 2. Baseline Jupiter gravity assist mission.

\section{Multi-planet Gravity Assist}

To complete the examination of the trajectory trade space, three multi-planet gravity assists were considered: Jupiter-Saturn gravity assist (JSGA), Jupiter-Uranus gravity assist (JUGA), and Jupiter-Neptune gravity assist (JNGA). Of these three options, only the JSGA gave a trip time below 30 years (23 years to $200 \mathrm{AU}$ with 20 years of EP thrusting). In contrast, the JUGA and JNGA launch opportunities (both in 2013) resulted in 56 and 65 year missions, respectively, with no significant mass reductions compared to even an SGA. The primary benefit of the JSGA is that it requires less propellant than the JGA ( $298 \mathrm{~kg}$ compared to the $394 \mathrm{~kg}$ for the JGA) at only a slightly higher $\mathrm{C}_{3}\left(160\right.$ versus $\left.152.6 \mathrm{~km}^{2} / \mathrm{s}^{2}\right)$. However, the (late) 2037 launch date does not make it a viable candidate for the current study.

\section{Jupiter Gravity Assist Launch Dates}

Other launch dates, ranging from 2010 to 2020 , for the JGA were also considered. These alternate launch opportunities are "backup" windows for the baseline 2014. The maximum trip time approached 55 years for a 2020 launch. Other window occur at roughly 13-month intervals (the Earth-Jupiter synodic period):

- launching in Aug 2012 would require 18.4 years of EP thrusting (832 kg propellant) for a 30.9-year trip;

- launching in Sep 2013 would require 16.1 years of EP thrusting (768 kg propellant) for a 27-year trip;

- launching in Nov 2015 would require 15.5 years of EP thrusting (360 kg propellant) for a 26 year trip; and,

- launching in December 2016 would require 17.7 years of EP thrusting (361 kg propellant) for a 30.2-year trip.

The other important point is that this pattern recurs roughly once every 12 years, $\sim$ Jupiter's orbital period about the Sun. Hence, during the 2010-2050 time frame, the JGA mission has four sets of launch opportunities. All other gravity-assist scenarios have only one launch opportunity.

\section{E. Upper Stage Trades}

In each of the scenarios discussed the launch assumed the use of a Delta IV Heavy carrying the spacecraft on a two-stage stack consisting of a Star 48 and a Star 37. Even with such a combination, an Atlas V 551 was judged to have inadequate capability to provide a self-consistent solution for the 519-kg dry spacecraft. As the spacecraft 
design and metrics evolved (and margins were included), even this combination proved inadequate. The nominal upper stage adopted for the baseline consisted of a stack using two Star 48A motors on a Delta IV Heavy launch vehicle. A Star 63/Star 37 combination was also considered, but a mission design based upon it was not fully optimized. In all of the cases considered, including the baseline, the probe plus upper stage stack comes off the Delta IV with a large positive $\mathrm{C}_{3}$.

\section{The Spacecraft}

The general spacecraft approach is to keep the probe as simple as possible commensurate with launch vehicle availability and the payload requirements. This led to the early investigation of how the instruments and their observing and communications requirements impacted the spacecraft and vice versa.

\section{A. Trades, Studies, and Risks}

All-sky coverage by fields and particles instruments is required. A spinning spacecraft provides coverage but must reorient for downlinks because the optimal thrust direction changes with respect to the direction toward the Earth. In addition, there is a need to minimize forces and torques on the (long) magnetometer boom and plasma wave antennas. The spin rate must be sufficiently fast to provide all sky covererage in azimuth on $\sim$ houly to $\sim$ daily timescales.

1. Communcations

The spin rate must also provide for downlink communications stability. An optical downlink requires submicroradian pointing at $200 \mathrm{AU}$. This accuracy cannot be accommodated on "small" spacecraft, so Ka-band is baselined for the data downlink. Even for this larger signal spread, the high-gain antenna must be hard mounted to the spacecraft bus to enable the required pointing accuracy at 200 AU. Throughout, this study, achieving communications from deep space at an adequate bandwidth was the main, non-propulsion system driver.

The conflict between thrust orientation and downlink direction (to Earth) means there is a trade between total data volume downlinked and powered flight. This trade also includes mission operations costs (for frequent downlink periods) versus autonomy (for rare downlink periods). In order to deal with the conflicting pointing requirements, for downlink periods the procedure is: turn off engine, reorient spacecraft, receive lock signal (prebroadcast) to verify pointing (200 AU is $\sim 27$ light-hours), downlink data obtained since last contact, reorient for thrust, and turn engine on until next downlink. All mission design scenarios have the termination of thrusting at $\sim 100$ AU with cruise thereafter. So, at end of thrusting (all Xe used), the spacecraft can be permanently oriented toward Earth. This introduces power cycling on the engine and requires more guidance and control consumables during thrust period. On the other hand, this approach also frees up thruster power for communication system use during downlink. It also requires autonomous spacecraft orientation for downlink and some autonmomy to respond to emergency-mode uplink (if the spacecraft misses a downlink session). Hence, the entire communications requirement impacts autonomy, guidance and control - and associated consumables - as well as stability and highgain antenna size trades versus the downlink communication power system hardware (and associated mass).

\section{System Mass}

Some of the initial mission design trade studies show there is a direct scaling between the flyout time and dry mass of the system for a fixed power input (Figure 3). To minimize the mass and match the optimized mission design specific impulse, a low mass, high-efficiency, system with high throughput and reliability is required. The mission design needs specific impulse in the range of $\sim 3600$ s to $4400 \mathrm{~s}$, so we baseline ion thrusters. Thruster operation below $\sim 750 \mathrm{~W}$ is not efficient, so the approach is have one thruster at a time to operate at the highest power level consistent with power availability. To minimize propulsion system mass we keep the system as simple as possible and use supercrtitical $\mathrm{Xe}$ as propellant. Thruster power is limited by required power for the spacecraft and instrument operational needs and the end-of-life power output, so

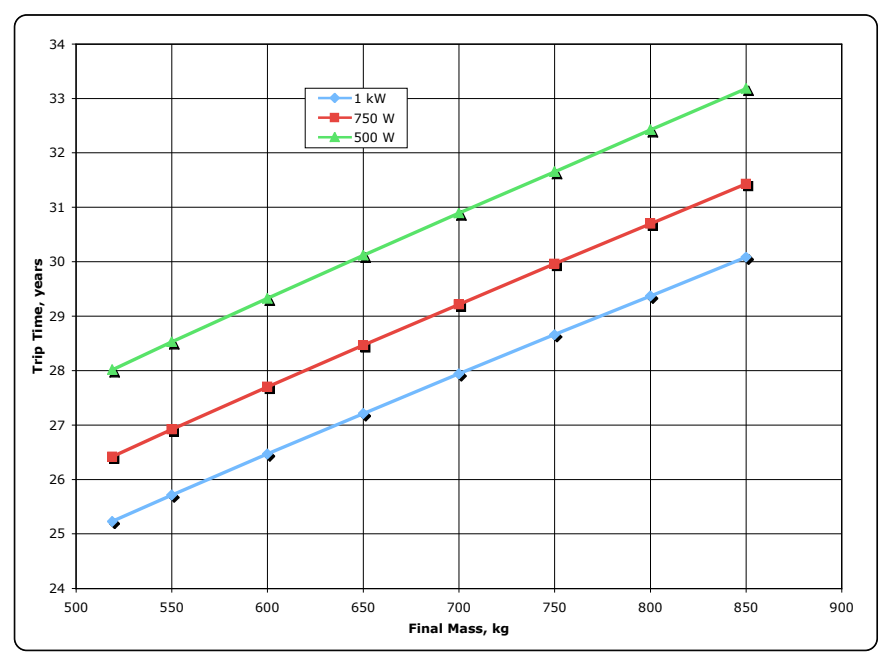

Figure 3. Trip time to $200 \mathrm{AU}$ as a function of final (dry) mass for fixed engine power levels. 
operational spacecraft and instrument power need to be minimized as well. In addition, multiple ion engines must be flown for reliability as well as their qualified mass processing capabilities. All of these trades including consideration of avionics and power processing unit mass must be taken into consideration in the final design.

\section{Thrusting and Data Collection}

Plasma wave and magnetometer data are critical for mission success but are also interfered with (at some level) by ion engine operation. In addition, Stirling radioisotope generators, a choice for the power supply that helps minimize the required $\mathrm{Pu}-238$ has moving magnets that may also provide interference. For the magnetometer, we can baseline the dual inboard and outboard approach implemented on Voyager. This approach, along with the spacecraft spin will help calibrate out background fields. While we can limit data collection to times that the engines are off for downlink as a last resort, such an approach is not preferred. Development of electromagnetically "clean" ion engines and power supplies is a significant issue for proper implementation of this mission.

\section{Thruster Mounting}

Multiple thrusters are required that can only operate one at a time. Efficient operation requires thrust through the center-of mass, but the spacecraft is spinning and the high gain antenna must also be mounted near the center of mass to provide pointing accuracy With a sufficiently fast spin period and off-axis thrusters, the off-axis thrust torque will average out. This approach complicates the guidance and control system, and autonomy is made more critical during thrust period and especially during reorientation before and after downlink. Hence the efficiency of thrusting trades against pointing accuracy for the communications link at the systems level for realistic packaging configurations.

\section{Guidance and Control Functionality}

Gimbaling of the main ion thruster(s) is not sufficient to control spacecraft spin and downlink pointing scenarios. We have, therefore, baselined hydrazine thrusters to control the spacecraft attitude. In spite of obvious disadvantages, another consumable, another propulsion system, catbed heaters and required power - this approach still appears to be the lowest mass solution. Pulsed plasma thrusters are mass prohibitive with current and nearfuture implementations, and reaction wheels do not have sufficient lifetime, while also being massive and power hungry.

\section{B. Study Options}

Four options were investigated during the study. All options used the same architecture configuration, subsystem design, and baseline (JGA) mission design. Different technology and data rate assumptions drove the design of the 4 options. The baseline design (Option 1) relies on current state-of-the-art technology and does not make any aggressive technology assumptions, except for the power system - an advanced, high temperature RTG. A downlink data rate of $5.8 \mathrm{kbps}$ from $200 \mathrm{AU}$ is assumed (sufficient to collect downlinked data at a $500 \mathrm{bps}$ rate continuously) with two downlinks of $\sim 7$ hours per week to 180 phased 12-m antennas operating at Ka-band. The spacecraft has a $2.1-\mathrm{m}$ diameter high gain antenna and carries three $1-\mathrm{kW}$ ion engines, one being a spare. Four, fully redundant command and data subsystems (CDS) are used to deal with reliability questions for a $\sim 30$-yr flight time. This baseline design results in a system launch capability of 6,900 $\mathrm{kg}$ (includes Star 48 motors) after the EP trajectory was optimized, yielding a total trip time of 31.1 years to $200 \mathrm{AU}$.

The second study option is a delta from the baseline that investigates more aggressive technology and redundancy assumptions. Only two CDS strings and two engines are included. The high-gain antenna is increased to a 3-m diameter to compensate for other (mass and power reducing) system changes. The resulting design produces a slightly shorter trip time of 29.7 years to $200 \mathrm{AU}$ and a launch capability of $6,803 \mathrm{~kg}$.

Option 3 studies whether reducing the return data rate to $500 \mathrm{bps}$ (from $5.8 \mathrm{kbps}$ in Options 1 and 2) saves significant mass and power and, hence, reduces trip time. After optimization of the trajectory, the decreased data rate only saves around $20 \mathrm{~kg}$ of dry mass on the spacecraft from the baseline design.

Option 4 combines the aggressive technology in Option 2, the reduced data rate of Option 3, and a reduced ion engine power that ultimately results in a 29.9-year trip time and dry mass $170 \mathrm{~kg}$ less than that of the baseline design. The high gain antenna is $2.1-\mathrm{m}$ in diameter, 2 CDS strings are used, and two $750 \mathrm{~W}$ ion engines are used for propulsion.

In each case, power requirements for six operational power modes were evaluated and design reserves/margins were applied in accord with the technology readiness levels (TRLs) and design rules used by JPL's Team-X. Also, the overall mission design was re-optimized in each case assuming a "best launch date" in 2014 and a Jupiter gravity assist. Details for each option at the system level are given in Table 3. Corresponding optimized mission design details (assuming a Jupiter gravity assist) are given in Table 4. 
Table 3. Option Trades for Spacecraft System Design

\begin{tabular}{|c|c|c|c|c|c|c|c|c|c|c|c|c|}
\hline & $\begin{array}{l}\text { Option } \\
1 \\
\text { Baseline }\end{array}$ & $\begin{array}{l}\text { Option } 2 \\
\text { Aggressive } \\
\text { Technology } \\
\text { Assumptions }\end{array}$ & $\begin{array}{l}\text { Option } 3 \\
\text { Reduced } \\
\text { Downlink } \\
\text { Rate }\end{array}$ & $\begin{array}{l}\text { Option } 4 \\
\text { Aggressive } \\
\text { Technology } \\
\text { and } \\
\text { Reduced } \\
\text { Downlink } \\
\text { Rate }\end{array}$ & Subsys & CBE+ & $\begin{array}{l}\text { Mode } \\
1 \\
\text { Safing }\end{array}$ & $\begin{array}{l}\text { Mode } 2 \\
\text { Telecom } \\
\text { beyond } \\
103 \text { AU ( } 2 \\
8 \mathrm{hr} \text { passes } \\
\text { per week } \\
\text { at } 200 \mathrm{AU} \\
- \text { no no } \\
\text { thrusting, } \\
\text { continuous } \\
\text { science) }\end{array}$ & $\begin{array}{l}\text { Mode } 3 \\
\text { Engine-off } \\
\text { Cruise 103- } \\
200 A U \\
\text { (Continuous } \\
\text { Science) }\end{array}$ & $\begin{array}{l}\text { Mode } 4 \\
\text { Engine-on } \\
\text { Cruise 10 to } \\
103 \text { AU } \\
\text { (Continuous } \\
\text { Science) }\end{array}$ & $\begin{array}{l}\text { Mode } 5 \\
\text { Launch }\end{array}$ & $\begin{array}{l}\text { Mode } 6 \\
\text { Telecom } \\
\text { to } 103 \mathrm{AU} \\
\text { (One } 8 \mathrm{hr} \\
\text { pass per } \\
\text { week -ek } \\
\text { continuous } \\
\text { science, } \\
\text { no } \\
\text { thrusting } \\
\text { during } \\
\text { telecom) }\end{array}$ \\
\hline & $\begin{array}{l}\text { Mass } \\
(\mathrm{kg})\end{array}$ & Mass $(\mathrm{kg})$ & Mass $(\mathrm{kg})$ & Mass $(\mathrm{kg})$ & $\begin{array}{l}\begin{array}{l}\text { Cont. } \\
(\%)\end{array} \\
\end{array}$ & $\begin{array}{l}\text { Cont. } \\
(\mathrm{kg})\end{array}$ & $\begin{array}{l}\text { Pwr } \\
\text { (W) }\end{array}$ & Pwr (W) & Pwr (W) & Pwr (W) & Pwr (W) & Pwr (W) \\
\hline \multicolumn{13}{|l|}{ Payload } \\
\hline Instruments & 35.2 & 35.2 & 35.2 & 35.2 & $30 \%$ & 45.7 & 9.1 & 29.4 & 29.4 & 29.4 & 0.0 & 29.4 \\
\hline $\begin{array}{l}\text { Payload } \\
\text { Total }\end{array}$ & 35.2 & 35.2 & 35.2 & 35.2 & $30 \%$ & 45.7 & 9.1 & 29.4 & 29.4 & 29.4 & 0.0 & 29.4 \\
\hline \multicolumn{13}{|l|}{ Bus } \\
\hline $\begin{array}{l}\text { Attitude } \\
\text { Control }\end{array}$ & 14.9 & 6.8 & 14.9 & 6.8 & $21 \%$ & 18.0 & 9.0 & 36.0 & 36.0 & 36.0 & 40.0 & 36.0 \\
\hline $\begin{array}{l}\text { Command \& } \\
\text { Data }\end{array}$ & 25.8 & 13.9 & 25.8 & 13.9 & $30 \%$ & 33.5 & 43.0 & 43.0 & 43.0 & 43.0 & 43.0 & 43.0 \\
\hline Power & 182.3 & 182.3 & 182.3 & 154.6 & $30 \%$ & 237.0 & 10.1 & 46.0 & 10.4 & 11.4 & 8.2 & 46.5 \\
\hline Propulsion1 & 80.9 & 61.7 & 80.9 & 59.0 & $20 \%$ & 97.1 & 0.7 & 0.7 & 0.7 & 0.7 & 0.7 & 0.7 \\
\hline Propulsion2 & 10.4 & 10.4 & 10.4 & 10.3 & $18 \%$ & 12.3 & 41.0 & 41.0 & 1.0 & 1.0 & 1.0 & 41.0 \\
\hline $\begin{array}{l}\text { Structures } \\
\& \\
\text { Mechanisms }\end{array}$ & 126.5 & 109.6 & 124.0 & 99.0 & $30 \%$ & 164.4 & 0.0 & 0.0 & 0.0 & 0.0 & 0.0 & 0.0 \\
\hline $\begin{array}{l}\text { S/C-side } \\
\text { Adapter }\end{array}$ & 0.0 & 0.0 & 0.0 & 0.0 & $30 \%$ & 0.0 & & & & & & \\
\hline Cabling & 37.9 & 30.4 & 37.2 & 28.2 & $30 \%$ & 49.3 & & & & & & \\
\hline Telecomm & 23.1 & 24.2 & 21.1 & 21.1 & $20 \%$ & 27.7 & 17.0 & 522.6 & 17.0 & 17.0 & 17.0 & 517.0 \\
\hline Thermal & 47.7 & 41.7 & 38.5 & 34.7 & $30 \%$ & 61.9 & 34.5 & 34.5 & 32.0 & 47.5 & 23.8 & 47.5 \\
\hline $\begin{array}{l}\text { Bus } \\
\text { Total } \\
\end{array}$ & 549.5 & 481.0 & 534.9 & 427.7 & $28 \%$ & 701.1 & 155.2 & 723.8 & 140.0 & 156.5 & 133.6 & 731.6 \\
\hline $\begin{array}{l}\text { Spacecraft } \\
\text { Total } \\
\text { (Dry) } \\
\end{array}$ & 584.6 & 516.2 & 570.1 & 462.8 & $28 \%$ & 746.9 & 164.3 & 753.2 & 169.4 & 185.9 & 133.6 & 761.0 \\
\hline $\begin{array}{l}\text { Subsystem } \\
\text { Heritage } \\
\text { Contingency }\end{array}$ & 162.2 & 144.6 & 158.1 & 128.9 & $28 \%$ & $28 \%$ & & & & & & \\
\hline $\begin{array}{l}\text { System } \\
\text { Contingency }\end{array}$ & 13.2 & 10.3 & 13.0 & 10.0 & $2 \%$ & $2 \%$ & 49.3 & 225.9 & 50.8 & 55.8 & 40.1 & 228.3 \\
\hline $\begin{array}{l}\text { Spacecraft } \\
\text { with } \\
\text { Contingency }\end{array}$ & 760.0 & 671.1 & 741.1 & 601.7 & & & 213.6 & 979.1 & 220.3 & 241.7 & 173.6 & 989.4 \\
\hline $\begin{array}{l}\mathrm{Xe} \\
\text { Propellant \& } \\
\text { Pressurant 1 }\end{array}$ & 459.4 & 449.6 & 460.8 & 393.8 & & & & & & & & \\
\hline $\begin{array}{l}\text { Hydrazine } \\
\text { Propellant \& } \\
\text { Pressurant2 }\end{array}$ & 30.5 & 30.5 & 30.5 & 30.5 & & & & & & & & \\
\hline $\begin{array}{l}\text { Spacecraft } \\
\text { Total } \\
\text { (Wet) }\end{array}$ & 1249.9 & 1151.2 & 1232.5 & 1026.0 & & & & & & & & \\
\hline
\end{tabular}

\section{Enabling Technologies and Infrastructure}

\section{Power System}

In each case power and propulsion are, not surprisingly, major contributors to the overall spacecraft dry mass. In particular, high power-to-mass radioactive power sources (RPS) are the key enabling technology. Currently flying RTGs and the proposed MMRTG have power-to-mass ratios that are too small for this type of mission. Possibilities for adequate $(>8 \mathrm{~W} / \mathrm{kg})$ RPSs include "second generation" Stirling Radioisotope Generators (SRGs) and hightemperature, high-efficiency RTGs. Both approaches have pluses and minuses and both need further development. SRGs hold the promise of high-efficiency conversion that lowers the need for Pu-238 (the optimum radioisotope for long-term deep-space-qualified power supplies). Issues are electromagnetic interference (EMI) with instruments, here notable the magnetometer and plasma wave sensor, and lifetime of the generator due to wear of the moving parts. Advanced high-temperature RTGs using, e.g. skutterudite converters, hold promise for high-efficiency and long lifetime with no EMI (no moving parts) but have inherently lower conversions efficiencies. Lowered efficiency equates to a need of $\sim$ four times as much $\mathrm{Pu}-238$ as SRGs, a significant cost, safety, and risk issue. These issues also apply to all other small deep-space (beyond the orbit of Jupiter) probes and warrant technical closure as soon as possible. 
Table 4. Optimized Mission Designs (JGA) for Each Architecture Option

\begin{tabular}{|c|c|c|c|c|}
\hline & $\begin{array}{l}\text { Option } 1 \\
\text { Baseline }\end{array}$ & $\begin{array}{ll}\text { Option } 2 & \\
\text { Aggressive } \\
\text { Assumptions }\end{array}$ Technology & $\begin{array}{l}\text { Option } 3 \\
\text { Reduced Downlink Rate }\end{array}$ & 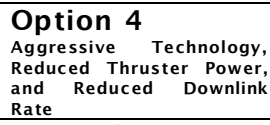 \\
\hline Spacecraft Total (Dry) & $584.6 \mathrm{~kg}$ & $516.2 \mathrm{~kg}$ & $570.1 \mathrm{~kg}$ & $462.8 \mathrm{~kg}$ \\
\hline Subsystem Heritage Contingency & $162.2 \mathrm{~kg}$ & $144.6 \mathrm{~kg}$ & $158.1 \mathrm{~kg}$ & $128.9 \mathrm{~kg}$ \\
\hline System Contingency & $13.2 \mathrm{~kg}$ & $10.3 \mathrm{~kg}$ & $13.0 \mathrm{~kg}$ & $10.0 \mathrm{~kg}$ \\
\hline Spacecraft with Contingency & $760.0 \mathrm{~kg}$ & $671.1 \mathrm{~kg}$ & $741.1 \mathrm{~kg}$ & $601.7 \mathrm{~kg}$ \\
\hline Xe Propellant \& Pressurant & $459.4 \mathrm{~kg}$ & $449.6 \mathrm{~kg}$ & $460.8 \mathrm{~kg}$ & $393.8 \mathrm{~kg}$ \\
\hline Hydrazine Propellant \& Pressurant & $30.5 \mathrm{~kg}$ & $30.5 \mathrm{~kg}$ & $30.5 \mathrm{~kg}$ & $30.5 \mathrm{~kg}$ \\
\hline Spacecraft Total (Wet) & $1249.9 \mathrm{~kg}$ & $1151.2 \mathrm{~kg}$ & $1232.5 \mathrm{~kg}$ & $1026.0 \mathrm{~kg}$ \\
\hline 2 Star $48 \mathrm{~A}$ Motors with $2 \%$ contingency & $5265.2 \mathrm{~kg}$ & $5265.2 \mathrm{~kg}$ & $5265.2 \mathrm{~kg}$ & $5265.2 \mathrm{~kg}$ \\
\hline Adapter from top Star 48 to $\mathrm{s} / \mathrm{c} \mathrm{w} / 30 \%$ cont & $45.8 \mathrm{~kg}$ & $32.1 \mathrm{~kg}$ & $45.3 \mathrm{~kg}$ & $28.9 \mathrm{~kg}$ \\
\hline Adapter between 2 Star 48 Motors w/ 15\% & $209.3 \mathrm{~kg}$ & $209.3 \mathrm{~kg}$ & $209.3 \mathrm{~kg}$ & $209.3 \mathrm{~kg}$ \\
\hline Adapter from LV to bottom Star $48 \mathrm{w} / 15 \%$ & $104.7 \mathrm{~kg}$ & $104.7 \mathrm{~kg}$ & $104.7 \mathrm{~kg}$ & $104.7 \mathrm{~kg}$ \\
\hline Launch Mass & $6874.9 \mathrm{~kg}$ & $6762.4 \mathrm{~kg}$ & $6856.9 \mathrm{~kg}$ & $6634.1 \mathrm{~kg}$ \\
\hline Launch Vehicle Capability & $6906.0 \mathrm{~kg} \mathrm{~kg}$ & $6803.0 \mathrm{~kg}$ & $6887.0 \mathrm{~kg}$ & $6678.0 \mathrm{~kg}$ \\
\hline Margin & $31.1 \mathrm{~kg}$ & $40.6 \mathrm{~kg}$ & $30.1 \mathrm{~kg}$ & $43.9 \mathrm{~kg}$ \\
\hline Spacecraft Mass Margin (\%) & $0 \%$ & $1 \%$ & $0 \%$ & $1 \%$ \\
\hline Launch Date & October 22,2014 & October 23,2014 & October 22,2014 & October 24, 2014 \\
\hline Gravity Assist Body & Jupiter & Jupiter & Jupiter & Jupiter \\
\hline Gravity Assist Date & February 5, 2016 & January 21,2016 & February 2, 2016 & January 8,2016 \\
\hline Gravity Assist Altitude & $75150 \mathrm{~km}$ & $67658 \mathrm{~km}$ & $73695 \mathrm{~km}$ & $61904 \mathrm{~km}$ \\
\hline Gravity Assist Radius & $2.05 \mathrm{Rj}$ & $1.95 \mathrm{Rj}$ & $2.03 \mathrm{Rj}$ & $1.87 \mathrm{Rj}$ \\
\hline Gravity Assist $\Delta \mathrm{v}$ & $23.8 \mathrm{~km} / \mathrm{s}$ & $24.8 \mathrm{~km} / \mathrm{s}$ & $24.0 \mathrm{~km} / \mathrm{s}$ & $25.5 \mathrm{~km} / \mathrm{s}$ \\
\hline Burnout Date & October 13,2032 & December 4, 2031 & August 9, 2032 & April 10,2032 \\
\hline Burnout Distance & $104 \mathrm{AU}$ & $104 \mathrm{AU}$ & $104 \mathrm{AU}$ & $106 \mathrm{AU}$ \\
\hline Burnout Speed & $7.9 \mathrm{AU} /$ year & $8.3 \mathrm{AU} /$ year & 7.9 AU/year & $8.1 \mathrm{AU} /$ year \\
\hline Date 200 AU Reached & December 31, 2044 & July 24,2043 & September 12, 2044 & October 31,2043 \\
\hline Minimum Trip Time to $200 \mathrm{AU}$ & 30.2 years & 28.8 years & 29.9 years & 29.0 years \\
\hline Speed at $200 \mathrm{AU}$ & $7.8 \mathrm{AU} / \mathrm{yr}$ & $8.3 \mathrm{AU} / \mathrm{yr}$ & $7.9 \mathrm{AU} / \mathrm{yr}$ & $8.1 \mathrm{AU} / \mathrm{yr}$ \\
\hline Right Ascension at $200 \mathrm{AU}$ & $263.8^{\circ}$ & $261.5^{\circ}$ & $263.4^{\circ}$ & $259.9^{\circ}$ \\
\hline Declination at $200 \mathrm{AU}$ & $0.0^{\circ}$ & $0.0^{\circ}$ & $0.0^{\circ}$ & $0.0^{\circ}$ \\
\hline Launch Mass & $1230 \mathrm{~kg}$ & $1135 \mathrm{~kg}$ & $1210 \mathrm{~kg}$ & $1013 \mathrm{~kg}$ \\
\hline Propellant Mass & $440 \mathrm{~kg}$ & $433 \mathrm{~kg}$ & $439 \mathrm{~kg}$ & $380 \mathrm{~kg}$ \\
\hline Final Mass (=S/C w/contingency+margin) & $790 \mathrm{~kg}$ & $702 \mathrm{~kg}$ & $771 \mathrm{~kg}$ & $633 \mathrm{~kg}$ \\
\hline Power & $1.0 \mathrm{~kW}$ & $1.0 \mathrm{~kW}$ & $1.0 \mathrm{~kW}$ & $0.75 \mathrm{~kW}$ \\
\hline$I_{s p}$ & $3800 \mathrm{~s}$ & $3734 \mathrm{~s}$ & $3784 \mathrm{~s}$ & $3479 \mathrm{~s}$ \\
\hline EP System Efficiency & $53.8 \%$ & $53.8 \%$ & $53.8 \%$ & $53.5 \%$ \\
\hline Total Stack $\mathrm{C}_{3}$ & $123.3 \mathrm{~km}^{2} / \mathrm{s}^{2}$ & $129.0 \mathrm{~km}^{2} / \mathrm{s}^{2}$ & $124.3 \mathrm{~km}^{2} / \mathrm{s}^{2}$ & $136.0 \mathrm{~km}^{2} / \mathrm{s}^{2}$ \\
\hline Delta IV $\mathrm{H} \mathrm{C}_{3}$ & $16.1 \mathrm{~km}^{2} / \mathrm{s}^{2}$ & $17.6 \mathrm{~km}^{2} / \mathrm{s}^{2}$ & $16.9 \mathrm{~km}^{2} / \mathrm{s}^{2}$ & $18.5 \mathrm{~km}^{2} / \mathrm{s}^{2}$ \\
\hline Delta IV H Launch Mass & $6938 \mathrm{~kg}$ & $6743 \mathrm{~kg}$ & $6832 \mathrm{~kg}$ & $6622 \mathrm{~kg}$ \\
\hline EP $\Delta v$ & $16.5 \mathrm{~km} / \mathrm{s}$ & $17.6 \mathrm{~km} / \mathrm{s}$ & $16.7 \mathrm{~km} / \mathrm{s}$ & $16.1 \mathrm{~km} / \mathrm{s}$ \\
\hline
\end{tabular}

\section{Ion Engine}

While electrostatic ion engines of nearly the required specific impulse ( $\sim 3800 \mathrm{~s})$ are currently available, mass of the engines and associated hardware is an issue. In particular, for REP-enable missions, high-efficiency, low power $(\sim 1 \mathrm{~kW})$ thusters that are space-qualified are needed. Xenon is the propellant of choice due to its easier storage than kypton or argon. Missions such as this with relatively low Xe needs $(\sim 500 \mathrm{~kg})$ can easily deal with the relatively high cost of xenon (world-wide Xe production is $\sim 6$ x $10^{6}$ liters $/ \mathrm{yr}$ or $35,000 \mathrm{~kg} / \mathrm{yr}$ currently). The power-processing unit (PPU) is a relatively heavy component in an EP system. Typically, such units are made to be throttled over a wide dynamic range for use in solar electric propulsion applications. For REP, the only variance in power level is due to the radioactive decay of the plutonium and the degradation of the converter. Hence, less dynamic range is needed and this could lead to a more optimized design.

3. Communications

We have previously noted that for a small platform, Ka-band offers advantages over optical communications due to the pointing tolerances on the spacecraft. This choice, along with the limited onboard power supply favors larger receiving antennas on the ground. An excellent communications downlink can be achieved with the planned Deep Space Network (DSN) upgrade to a massively phased array of $\sim 12-\mathrm{m}$ dishes. The trade is against anticipated operating cost of such an array that can provide a significant mission cost over a 30 -year mission. 


\section{Expendable Launch Vehicles}

All REP schemes to the outer solar system and beyond require large launch energies. To enable such missions in general, and this one in particular, large launch vehicles (such as the Delta IVH) and custom upper stages that can be qualified for use with advanced RPS systems must be available in the time frame of the mission development and launch (here 2010-2015).

5. Long-term Qualified Parts and Subsystems

Missions that have a planned long lifetime require parts and subsystems that can be qualified to some multiple of that interval without requiring real-time testing. For a 30-year mission launching in 2014, real-time testing would have had to commence earlier than 1985 . While subtle, this qualification problem may be the most difficult to deal with to the satisfaction of everyone with a stake in such a long-term robotic mission.

\section{Spacecraft Configuration}

Some sketches of the spacecraft configuration are shown in Figure 4. Here Option 2 is depicted (3-m diameter high-gain antenna and two thrusters). Note that both the thrusters and high-gain antenna are off axis from the spin axis. The bus, xenon tank, and six RPS units are all symmetric about the spin axis. The three plasma-wave antennas are orthogonal to each other and face toward the direction of motion and away from the active thruster. The size and mass of the dry spacecraft is intermediate to that of the Pioneer 10/11 spacecraft and the Voyager spacecraft.

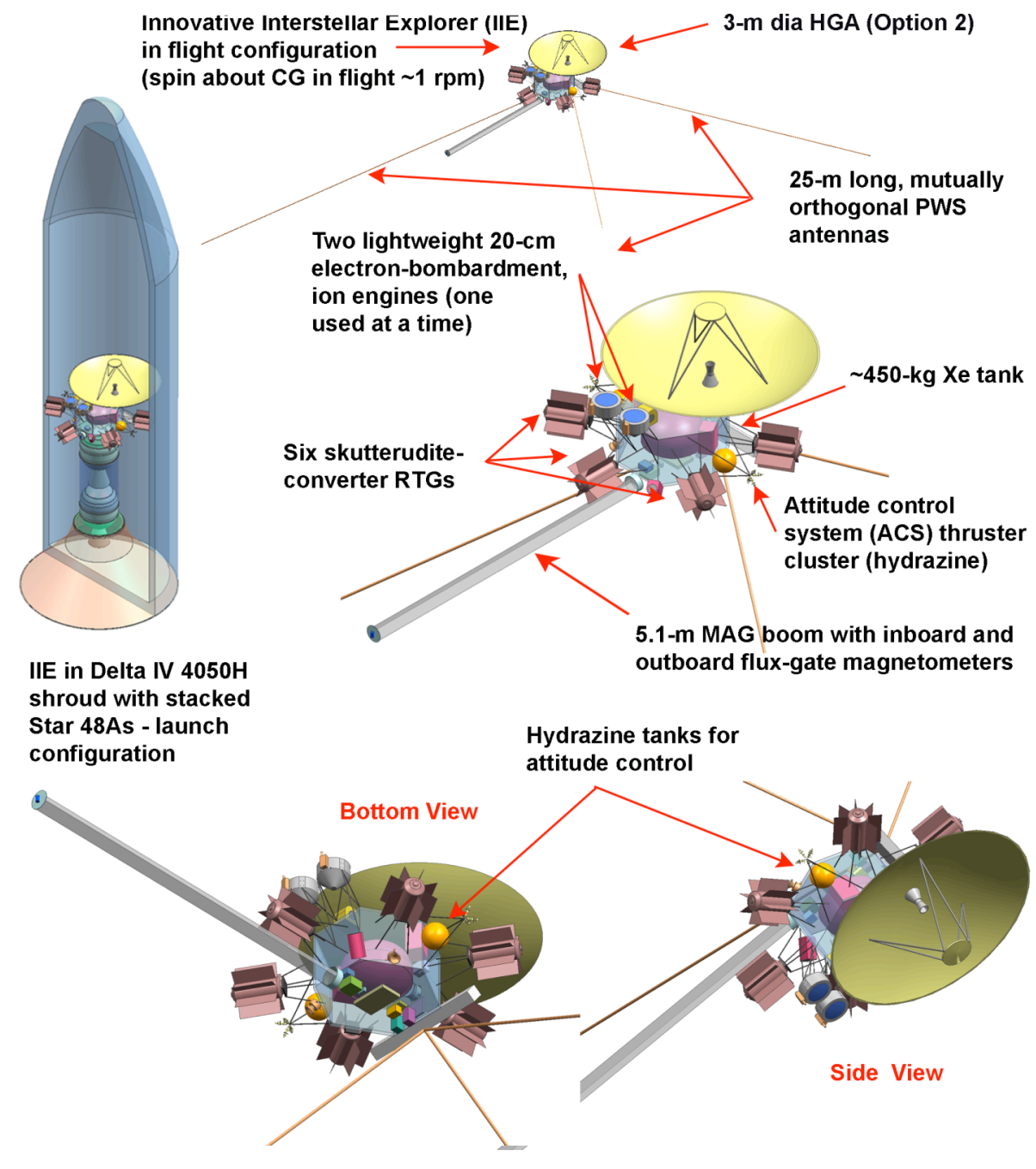

Figure 4. Isometric views of the Innovative Interstellar Explorer spacecraft. 


\section{Implementation}

The next launch window in 2014 for a mission to the interstellar medium is not that far off. While there is a "season" of launch possibilities that extends to early 2018, missing this window would delay the launch of such a mission until at least 2026. While the Jupiter flyby is a significant constraint on these possibilities, the gain due to a flyby of Jupiter is too great to be ignored: $\sim 25 \mathrm{~km} / \mathrm{s}$ or $5.3 \mathrm{AU} / \mathrm{yr}$ for each of the options considered (see Table 4). The use of Jupiter to reach an aim-point near the heliospheric "nose," i.e. a closest approach to the interstellar medium, dictates possible launch years.

For a launch in 2014, the technology must be in place, i.e. tested by 2010. In addition, by that time, radioisotope fuel procurement would need to begin along with the various studies and approvals required by the National Environmental Policy Act (NEPA) as pertaining to the launch of nuclear materials.

For our option 1, a launch on October 22, 2014 leads to a Jupiter flyby about one Jovian radius above the planet's cloud tops on February 5, 2016. The spacecraft begins routine heliospheric data collection soon after this date. By six years into the flight (2020), the Voyager 1 and 2 spacecraft will cease transmission, even with power sharing. Voyager 1 will be $\sim 150$ AU from the Sun and Voyager $2 \sim 125$ AU from the Sun. They may have passed the heliopause (or what passes for it by this time); it is doubtful that they will have reached the undisturbed interstellar medium.

On October 13, 2032, IIE will have depleted all xenon propellant with a burnout speed of $7.9 \mathrm{AU} / \mathrm{yr}$ and now $104 \mathrm{AU}$ from the Sun. The spacecraft will now be in the vicinity of the termination shock structure passed by Voyager 1 late in 2004. IIE be 18 years from launch; reaching this distance will take Voyager 1 about 30 years (it is not quite there yet). IIE will reach 200 AU on New Year's Eve of 2044, just over 30 years after launch and still coasting at $\sim 7.8 \mathrm{AU} / \mathrm{yr}$. By 2057, some 43 years after launch, the spacecraft would pass 300AU. This is the length of time from launch that the Voyagers are expected to finally go silent.

For IIE, there will be plenty of power left as the Pu-238 fuel in the six RTGs continues to decay. Continued tracking will depend upon how well the high-gain antenna can be pointed and the distance radio signal acquired. In the year 2147, over a century after launch, IIE will be the fastest - and farthest - manufactured object produced by humanity as it passes the1000-AU mark from the Sun.

\section{Conclusion}

A space probe to the interstellar medium has proven elusive due to the substantial distances involved. A realistic mission that has some hope of being implemented must have a planned mission length measured in decades at the most. Robotic missions must rely upon proven technology and launch vehicles. Evolutionary advance of some systems may be considered on anew mission, but only as long as the developmental risks appear to be low. Against this backdrop, a scientific requirement for such a mission has been articulated and advocated for 30 years. Propulsion has always been the driving problem. Previous studies have considered ballistic near-Sun gravity assists, large NEP systems, and small solar-sail driven systems. In each case, the propulsion technology has been - and remains - just out of reach. We have provided a first cut of a self-consistent design for such a mission using REP, existing launch vehicle hardware, and a Jupiter gravity assist. While the asymptotic speed of the probe is not as high as might be wished, it is sufficiently high to provided new - and potentially transformational - knowledge of our surroundings in interstellar space. More importantly, the required technology advances are evolutionary such that the probe could be built - and launched - as soon as the next launch window opens in late 2014.

\section{Acknowledgments}

The work was supported by NASA "Vision Mission" grant NNG04GJ60G. We acknowledge contributions of NASA Jet Propulsion Laboratory's Team-X. The views expressed herein are not necessarily endorsed by the sponsor.

\section{References}

${ }^{1}$ McNutt, Jr., R. L., Lyon, J., and Goodrich, C. C., “Simulation of the heliosphere: Model,” J. Geophys. Res., Vol. 103, 1998, pp. 1905-1912.

${ }^{2}$ McNutt, Jr., R. L., Lyon, J. , and Goodrich, C. C., "3D MHD Simulations of the Heliosphere-VLISM Interaction,” AIP Conf. Proc. (Solar Wind 9), Vol. CP471, 1999, pp. 823-826.

${ }^{3}$ McNutt, Jr., R. L., Lyon, J., and Goodrich, C. C., "Simulation of the heliosphere: Generalized charge-exchange cross sections," J. Geophys. Res, Vol. 104, 1999, pp. 14803-14809. 
${ }^{4}$ McNutt, R. L., Jr., "Fluid modeling of the VLISM/solar wind interaction with the 13-moment formalism," in $A I P$ Conference Proceedings (Solar Wind 10), edited by M. Velli et al., Vol. 679, New York: American Institute of Physics, 2003, pp. 194-197.

${ }^{5}$ McNutt, R. L., Jr., "Modeling charge exchange in the solar wind/VLISM interaction," Physics of the Outer Heliosphere, Third International IGPP Conf., Florinski, V., Pogorelov, N. V., and G. P. Zank, eds., Vol. CP719, 2004, pp. 111-116.

${ }^{6}$ Krimigis, S. M., Decker, R. B., Hill, M. E., Armstrong, T. P., Gloeckler, G., Hamilton, D. C., Lanzerotti, L. J., and Roelof, E. C., "Voyager 1 exited the solar wind at a distance $>85$ AU from the Sun," Nature, Vol., 426, 2003, pp. 45-48.

${ }^{7}$ Gruntman M., McNutt R. L., Jr., Gold, R. E., Krimigis, S. M., Roelof E. C., Gloeckler, G., Koehn, P. L., Kurth, W. S., and Oleson, S. R., "Innovative Interstellar Explorer," 55-th International Astronautical Congress, Vancouver, Canada, IAC-04IAA.3.6.4.07, 2004.

${ }^{8}$ McNutt, R. L., Jr., Gold, R. E., Roelof, E. C., Zanetti, L. J., Reynolds, E. L., Farquhar, R. W., Gurnett, D. A., and Kurth, W. S., "A sole/ad astra: From the Sun to the stars," J. Brit. Inter. Soc., Vol. 50, 1997, pp. 463-474.

${ }^{9}$ McNutt, R. L., Jr., Andrews, G. B. McAdams, J., Gold, R. E., Santo, A., Oursler, D., Heeres, K., Fraeman, M., and Williams, B., "A realistic interstellar probe," COSPAR Colloquium on The Outer Heliosphere: The Next Frontiers COSPAR Colloquia Series, Vol. 11, K. Scherer, H. Fichtner, H.-J. Fahr, and E. Marsch, eds., Pergamon Press, New York, 2002, Vol. 11, pp. 431-434.

${ }^{10}$ Lyman, R. W., Ewing, M. E. Krishnan, R. S. Lester, D. M. and McNutt, R. L., Jr., "Solar thermal propulsion for an interstellar probe," AIAA 2001-3377, 37th AIAA/ASME/SAE/ASE Joint propulsion Conference, Salt Lake City, Utah, July 8$11,2001$.

${ }^{11}$ McNutt, R. L., Jr., Andrews, G. B., McAdams, J. V., Gold, R. E., Santo, A. G., Ousler, D. A., Heeres, K. J., Fraeman, M. E., and Williams, B. D., "Low-cost interstellar probe," Acta Astron., Vol. 52, 2003, pp. 267-279.

${ }^{12}$ McNutt, R. L., Jr., Andrews, G. B., Gold, R.E., Santo, A. G., Bokulic, R. S., Boone, B. G., Haley, D. R., McAdams, J. V., Fraeman, M. E., Williams, B. D., Boyle, M. P., Lester, D., Lyman, R., Ewing, M., Krishnan, R., Read, D., Naes, L., McPherson, M., and Deters, R., "A realistic interstellar explorer," in AIP Conference Proceedings (Solar Wind 10), edited by M. Velli et al., Vol. 679, New York: American Institute of Physics, 2003, pp. 830-833.

${ }^{13}$ McAdams, J. V., and McNutt, R. L. Jr., "Ballistic Jupiter gravity-assist, perihelion- $\Delta \mathrm{V}$ trajectories for an interstellar explorer," J. Astron. Sci., Vol. 51, 2003, pp.179-193.

${ }^{14}$ McNutt, R. L., Jr., G. B., Andrews, G. B., Gold, R.E., Bokulic, R. S., Boone, B. G., Haley, D. R., McAdams, J. V., Williams, B. D., Boyle, M. P., Starstrom, G., Riggin, J, Lester, D., Lyman, R., Ewing, M., Krishnan, R., Read, D., Naes, L., McPherson, M., and Deters, R., "A realistic interstellar explorer," Adv. Space Res., Vol. 34, 2004, pp.192-197.

${ }^{15}$ Jaffe, L. D., and Ivie, C. V., "Science aspects of a mission beyond the planets," Icarus, Vol. 39, 1979, pp.486-494.

${ }^{16}$ Jones, R. M. and Sauer, C. G., "Nuclear electric propulsion missions," J. Brit. Int. Soc., Vol. 36, 1984, pp.395-400.

${ }^{17}$ Jaffe, L. D. and Norton, H. N., "A prelude to interstellar flight," Astro. Aero., Vol. 18, 1980, pp.38-44.

${ }^{18}$ Jaffe, L. D., Ivie, C., Lewis, J. C., Lipes, R., Norton, H. N., Stearns, J. W., Stimpson, L. D., and Weissman, P., “An interstellar precursor mission," J. Brit. Int.. Soc., Vol. 33, 1980, pp.3-26.

${ }^{19}$ Pawlik, E. V., and Phillips, W. M., “A nuclear electric propulsion vehicle for planetary exploration,” J. Spacecraft Rockets, Vol., 14, 1977, pp.518-525.

${ }^{20}$ Nock, K. T., "TAU - A mission to a thousand astronomical units," 19th AIAA/DGLR/JSASS International Electric Propulsion Conference, Colorado Springs, CO, AIAA-87-1049, May 11-13, 1987.

${ }^{21}$ Mewaldt, R. A., and Liewer, P. C., "An interstellar probe mission to the boundaries of the heliosphere and nearby interstellar space," AIAA-2000-5173, AIAA Space 2000, 19-21 September 2000, Long Beach, California.

${ }^{22}$ Mewaldt, R.A., and Liewer, P.A., "Scientific payload for an Interstellar probe mission," The Outer Heliosphere: The Next Frontier, Pergamon, 2001, pp.451-464.

${ }^{23}$ Liewer, P. C., Mewaldt, R. A., Ayon, J. A., Garner, C., Gavit, S., and Wallace, R. A., "Interstellar probe using a solar sail: Conceptual design and technological challenges," COSPAR Colloquium on The Outer Heliosphere: The Next Frontiers, COSPAR Colloquia Series, Vol. 11, K. Scherer, H. Fichtner, H.-J. Fahr, and E. Marsch, eds., Pergamon, New York, 2001, pp.411-420.

${ }^{24}$ Noble, R. J., "Radioisotope electric propulsion of sciencecraft to the outer solar system and near-interstellar space," Acta Astron., Vol. 44, 1999, pp.193-199. 